\title{
The influence of burnout on skills retention of junior doctors at Red Cross War Memorial Children's Hospital: A case study
}

\author{
J M Stodel, A Stewart-Smith
}

This study used the Maslach Burnout Inventory to evaluate the degree of burnout among junior doctors at Red Cross War Memorial Children's Hospital (RXH), Cape Town, and the influence thereof on the retention of valuable skills in the hospital. It further considered measures that could be taken to mitigate the causes of burnout, by means of qualitative methods.

Background. The research explores the significance of burnout and the role it plays in the retention of junior doctors at RXH. There has been an increase in the migration of medical doctors worldwide, with an exodus of doctors from South Africa. Along with the effects of HIV/AIDS, this places extra strain on those who remain.

Methodology. A two-part, mixed quantitative and qualitative study consisting of a validated measure, the Maslach Burnout Inventory, was sent to 39 junior doctors at RXH. Responses were received from 23 doctors (one of which was invalid), constituting a $60 \%$ response rate. The second part consisted of four semistructured interviews.

Results. Of the 22 respondents, $100 \%$ experienced a high degree of burnout on one of the three scales of burnout, namely emotional exhaustion, depersonalisation and reduced accomplishment. Of those surveyed, 95\% expressed an intention to leave RXH.

Conclusion. The degree of emotional exhaustion and depersonalisation experienced by the junior doctors at RXH was significantly higher than that in a normative sample of 1104 doctors. Recruitment, improved management and planning, increased support, mentorship and a more empathetic administration were some of the factors suggested to mitigate the burnout experienced by the junior doctors.

S Afr Med J 2011;101:115-118.
Junior doctors in South Africa work long hours. Many hospitals require them to work 30-hour shifts and little provision is made for illness, with colleagues having to cover for each other if a fellow junior doctor is sick. AIDS has resulted in increasing numbers of patient deaths, and a higher burden of disease. This has added to the workload, and in many cases also to the complexity of decisions. All the above factors have increased the burnout rate among junior doctors.

At the same time there has been a significant exodus of doctors from South Africa. ${ }^{1}$ These doctors tend to be young (under 42 years), male and not yet specialised. This exodus vastly increases the workload of the doctors who remain. Unless sufficient emphasis is placed on the effects of burnout and other causes of medical migration, and attempts are made to mitigate them, South Africa will continue to lose doctors to the global labour market, ultimately resulting in detrimental standards of medical care.

The purpose of the research was to measure burnout in junior doctors at Red Cross War Memorial Children's Hospital (RXH), Cape Town, through the administration of a standardised measure known as the Maslach Burnout Inventory (MBI), and then statistically compare their burnout with that in a normalised sample of doctors supplied by Maslach et al. in their research guide. ${ }^{2} \mathrm{~A}$ sample of the doctors at RXH was then interviewed to qualitatively assess what can be done to mitigate the burnout.

Graduate School of Business, University of Cape Town

J M Stodel, MB BCh, DCH (SA), MBA

A Stewart-Smith, BA (Hons) MA, PhD

\section{Methods}

The research was conducted in two sections.

The first section was quantitative in nature. The MBI was used to measure the level of burnout experienced by the junior doctors. The burnout scores were compared statistically to normative data of a sample $(N=1104)$ of doctors.

The second section of the research was qualitative in nature and consisted of four interviews. The interviewees comprised one senior house officer (SHO), one junior registrar and two senior registrars. The interviews were recorded and transcribed, and subsequently coded and analysed in order to provide suggestions of what can be done to mitigate burnout at $\mathrm{RXH}$.

\section{The Maslach Burnout Inventory}

Burnout is defined as the syndrome of emotional exhaustion, depersonalisation and reduced personal accomplishments. It should be viewed as a continuous variable and not as a dichotomous variable that is either present or absent. ${ }^{2}$

The MBI was initially designed for use in all categories of human service $^{3}$ and was first formulated in $1981 .^{2}$ It is both valid and consistent, having been used to study burnout across professions and cultures. ${ }^{3}$ The MBI has been tested extensively as a measure. Internal reliability was estimated using Cronbach's coefficient alpha $(N=1316)$. The reliability coefficients for the subscales were 0.90 for emotional exhaustion, 0.79 for depersonalisation and 0.71 for personal accomplishment, where any value greater than 0.70 suggests validity. Convergent and discriminant validity of the measure was also demonstrated. ${ }^{2}$

Emotional exhaustion is characterised by a lack of energy and a feeling that one's emotional resources have been depleted. Reduced personal accomplishment is characterised by low motivation and self-esteem, while depersonalisation results in detachment and callousness towards others. ${ }^{4}$ 


\section{Research design, data collection methods and research instruments}

The research design was cross-sectional in nature, as are most previous studies regarding burnout in the literature. ${ }^{5}$

The completion of an electronic questionnaire was performed on a voluntary and private basis, thereby minimising response bias. In addition, it was conducted through a third party, which ensured respondent confidentiality. Furthermore, the questionnaire was presented as a survey on skills retention and did not make any mention of burnout. This ensured that the respondents were not sensitised to the concept of burnout and did not tailor their responses in this regard. Only questionnaires that were completed in full were analysed. Partial answers were discarded to protect the validity of any correlations or assumptions made.

The second section of the research involved four interviews, which followed a semi-structured standardised format and were completed in person.

\section{Sampling}

The sample for section 1 of the research was derived from the population of junior doctors at RXH in November 2009. The relevant population for the time period concerned consisted of 24 registrars and 15 SHOs (a total of 39 junior doctors). An e-mail with a link to an electronic survey was sent to all the SHOs and registrars.

The questionnaires were sent out repeatedly via e-mail until $60 \%$ of the population had responded. This response rate represents a $90 \%$ confidence interval with a $10 \%$ margin of error.

\section{Results}

\section{Section 1}

The mean age of the respondents was 30 years (standard deviation (SD) 3.26 years), with a 3:1 female preponderance. There was a mean postgraduate experience of 5 years and 9 months (SD 2 years 9 months), and mean hours worked per week 62.4 (SD 12) hours. This suggests that the average junior doctor at RXH was a 30 -year-old woman, with almost 6 years' postgraduate experience, who worked approximately 62 hours a week. If $\mathrm{RXH}$ aims to attract and retain staff, allowances must be made for the fact that female employees might want to have a family. In a scarce-skills environment such as this, an organisation with progressive maternity and childcare policies is more likely to attract and retain the skills it requires.

Virtually all the respondents indicated an intention to leave at the end of their rotation (Fig. 1). This corresponds with the high levels of emotional exhaustion illustrated by the survey (Table I). Table I shows that there is a strong correlation between high levels of emotional exhaustion and intention to leave, while Fig. 1 strikingly illustrates that a significant problem exists regarding the retention of valuable skills.

All 22 respondents experienced burnout on one of the three subscales of burnout. Fig. 2 illustrates the percentage of junior doctors surveyed who experienced high, moderate or low degrees of burnout on each of the burnout subscales. Each subscale was statistically compared with a normative sample of 1104 doctors.

\section{Emotional exhaustion (emotional resources are 'used up')}

The score for emotional exhaustion can be categorised as follows: low $-\leq 18$; moderate $-19-26$; high $-\geq 27$. These scores represent the lower, middle and upper thirds of a normative sample of 1104 doctors, with a mean of 22.19 (SD 9.53). The mean score for emotional exhaustion in the sample of junior doctors at RXH was

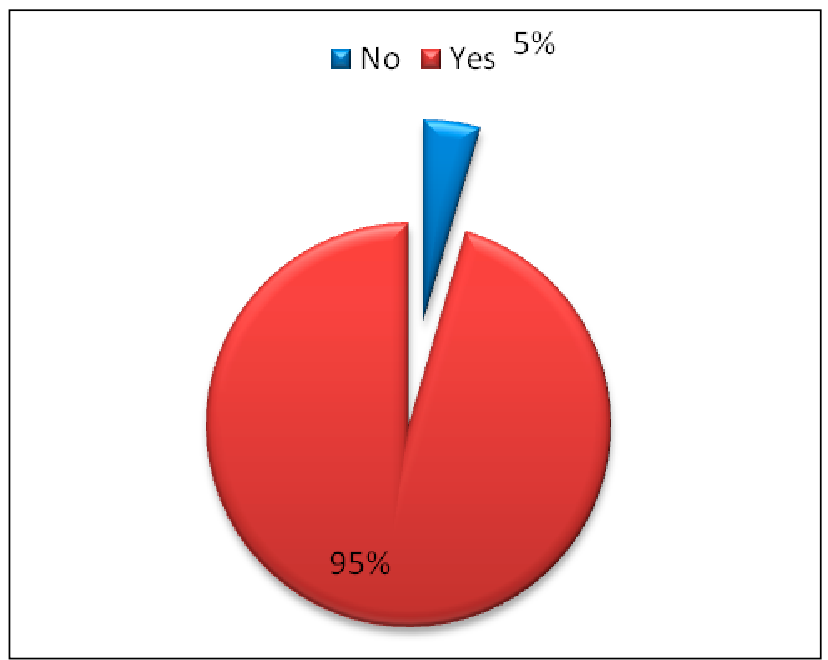

Fig. 1. Intention to leave Red Cross Hospital at the end of the subjects' rotation.

Table I. Relationship between emotional exhaustion and intention to leave

\begin{tabular}{llll}
\hline & \multicolumn{3}{c}{ Emotional exhaustion } \\
\cline { 2 - 4 } & High & Moderate & Total \\
\hline Leave & $19(86 \%)$ & $2(9 \%)$ & $21(95 \%)$ \\
Stay & $1(5 \%)$ & $0(0 \%)$ & $1(5 \%)$ \\
Total & $20(91 \%)$ & $2(9 \%)$ & $22(100 \%)$ \\
\hline
\end{tabular}

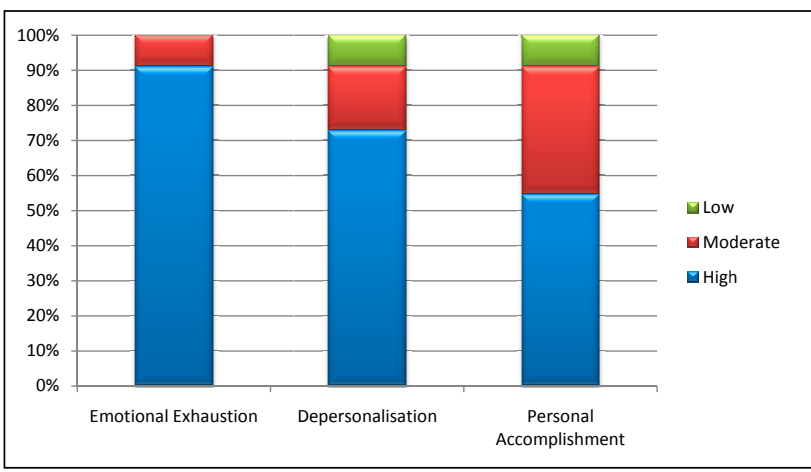

Fig. 2. Burnout experienced by junior doctors.

37.68 (SD 8.90). Levels of emotional exhaustion among the junior doctors at RXH are significantly higher $\left(p=3.29 \times 10^{-13}\right)$ than one would expect from a population of doctors when compared with the normative sample $(N=1104)$.

\section{Depersonalisation (detachment and callousness towards others)}

This score can be categorised as follows: low $-\leq 5$; moderate -6 - 9; high $-\geq 10$. The mean from the normative sample previously described is 7.12 (SD 5.22), while the mean score for this subscale of burnout for the RXH doctors was 12.63 (SD 5.60). There are significantly $\left(p=2.35 \times 10^{-7}\right)$ higher levels of depersonalisation at RXH than one would expect in a population of doctors when compared with the normative sample ( $N=1104)$. This is of particular concern as it implies that three-quarters of the junior doctors are detached and callous to their patients, which has implications for the quality of patient care. 
Reduced personal accomplishment (low motivation and self-esteem)

This score can be categorised as follows: low $-\geq 40$; moderate - 39 34 ; high $-\leq 33$. The mean from the normative sample previously described is 36.53 (SD 7.71). The mean score for the RXH doctors for this subscale of burnout was 32.14 (SD 5.83). It can be concluded that the junior doctors at $\mathrm{RXH}$ experience an average $(p=0.998)$ degree of personal accomplishment when compared with an average population of doctors.

\section{Recommendations from junior doctors}

Among the responses to the survey from the junior doctors, the most common recommendation for improvement for skills retention was revising the shift and leave system. This suggestion was strongly linked to the recruitment of additional staff (Table II).

\section{Section 2: analysis of interviews - causative and mitigating factors of burnout Factors that cause burnout (Table III)}

Workload was by far the most important factor contributing to burnout among the junior doctors. The doctors felt that because of workload there was not enough time to provide the level of care that they deemed necessary. This confirms previous research suggesting that role overload (i.e. workload) and role conflict (imposition of mutually incompatible expectations) are antecedents of burnout. Unrealistic workload expectations are associated with the second highest contributing factor - insufficient recruitment. ${ }^{6}$

The category 'poor planning' relates to administration foresight and the management of potential problems. These include both human resources management, such as pending maternity leave of colleagues, and operations management planning with regard to a potential influx of patients (as is typically the case during the annual gastro-enteritis season).

Emotional stress also added to the experience of burnout among junior doctors. This can be mitigated through mentorship, peer support groups, debriefing sessions and comprehensive new employee orientation programmes.

Table II. Recommendations from junior doctors for improving skills retention at Red Cross Hospital

\begin{tabular}{lcc}
\hline & $N$ & $\%$ \\
\hline Revising shift and leave system & 16 & 28 \\
Recruitment & 11 & 19 \\
Acknowledgement & 5 & 9 \\
Training & 5 & 9 \\
Supervisor input and support & 4 & 7 \\
Job sharing & 3 & 5 \\
Improve recreational facilities & 3 & 5 \\
Pay incentives & 2 & 3 \\
Improved leadership & 2 & 3 \\
Increased responsibilities of nursing and & 2 & 3 \\
senior staff & & \\
Enforcing referral system & 2 & 3 \\
Debriefing sessions & 2 & 3 \\
Improved collegiality & 1 & 2 \\
$\quad$ Total & 58 & 100 \\
\hline
\end{tabular}

Table III. Factors that cause burnout

\begin{tabular}{lll}
\hline & $N$ & $\%$ \\
\hline Workload & 40 & 26 \\
Insufficient recruitment & 30 & 19 \\
Emotional stress & 29 & 19 \\
Poor planning & 25 & 16 \\
Long hours & 25 & 16 \\
Lack of empathy from administration & 24 & 15 \\
No supervision/support & 16 & 10 \\
No mentoring & 15 & 10 \\
Poor training & 13 & 8 \\
No acknowledgement/appreciation & 7 & 4 \\
Inter-colleague stress & 5 & 3 \\
Effect on personal life & 4 & 3 \\
No job sharing & 2 & 1 \\
\hline
\end{tabular}

\section{Factors that mitigate burnout (Table IV)}

Analysis of the interviews suggests that a great deal of burnout experienced by the junior doctors could be mitigated through increased recruitment and improved management and planning by the administration. Increased support from senior colleagues, formal mentorship and an empathetic administration also featured highly as mitigating factors.

Formal mentorship and $360^{\circ}$ feedback sessions at the end of each junior doctor's block would motivate, encourage and support them.

\section{Discussion}

\section{Burnout and skills retention}

'Burnout results from prolonged high levels of stress at work and, if left untended, can contribute to the exodus of health care workers from these emotionally intense situations." Burnout is costly, not only in turnover but also with respect to patient care. Increased absenteeism and reduced productivity are common in areas with increased burnout. ${ }^{5}$ There is a direct correlation between burnout and quality of care: patients reported less satisfaction with the quality of care they received in units where the staff was burnt out. ${ }^{5}$

High employee turnover has grave implications for the quality, consistency and stability of services provided. High turnover rates

\section{Table IV. Factors that mitigate burnout}

\begin{tabular}{lcc}
\hline & $N$ & $\%$ \\
\hline Recruitment & 17 & 13 \\
Improved management/planning & 17 & 13 \\
Support & 15 & 12 \\
Mentorship & 14 & 11 \\
Empathetic administration & 14 & 11 \\
Improved staff relationships & 13 & 10 \\
Reduced hours & 13 & 10 \\
Emotional support & 10 & 8 \\
Acknowledgement & 9 & 7 \\
Improved training & 8 & 6 \\
\hline
\end{tabular}


can reinforce patients' mistrust of the system and can discourage health care workers from remaining in or even entering the field. 'Turnover can have detrimental effects on clients [and therefore patients] and remaining staff members who struggle to give and receive quality services when positions are vacated and then filled by inexperienced personnel. ${ }^{7}$

\section{Burnout in medical services}

Individuals who experience burnout perpetuate burnout in those around them. ${ }^{6}$ Furthermore, it is commonly accepted that high levels of stress in medical practice lead to increased risk of suicide, psychiatric morbidity, drug use and family disruption. ${ }^{6}$ 'Stress can be of utmost importance in a paediatric and neonatal intensive care unit ... as the interpersonal relations between staff and relatives can create dysphoric and depressive reactions in the professionals. ${ }^{7}$

Burnout has a direct dysfunctional influence on psychological performance and behavioural outcomes (job satisfaction, turnover intentions and job performance). In addition, 'burnout tendencies appear to significantly and positively affect turnover intentions. ${ }^{6}$ However, many employees feel a greater responsibility and commitment to their patients than they do towards their work organisation. This results in a conflict between the conditions (e.g. high caseloads) and the employees' own professional sense of duty, which results in some employees placing the needs of patients above their own emotional health. ${ }^{8}$

The issues of burnout and intention to leave are particularly salient in the health care industry, which is currently facing critical shortages of clinicians and difficulty in retaining these employees. Job stress has been shown to be positively associated with absenteeism and turnover among clinicians in general. ${ }^{9}$ There is evidence that workers typically make a conscious decision to quit long before doing so, and that this decision is the single strongest predictor of turnover. It is therefore more practical to measure intention to quit in a crosssectional study than to opt for a longitudinal study that tracks down workers who have already quit. ${ }^{10}$

\section{Burnout in South Africa}

Job stress is higher among South African physicians than physicians in Europe or the USA. The three greatest factors causing job stress among South African physicians are working overtime, making critical, on-the-spot decisions, and dealing with crisis situations. ${ }^{10}$

\section{What can be done to mitigate burnout?}

The existing literature suggests a number of interventions to mitigate burnout. Some are more applicable in resource-scarce South Africa than others. Mentoring programmes are recommended in order to provide a coping resource. Further recommendations include the suggestion that hospital managers find inventive ways to set shifts according to individual needs, as well as providing incentives to do unpopular shifts. The establishment of workload standards (e.g. maximum hours worked per day/week/month) is also suggested. 'Other steps associated with the amelioration of burnout may include improved recruitment and retention of new trainees and the introduction of productivity aides such as enhanced information systems. ${ }^{10}$

Expressing appreciation for the clinician as a cherished resource may help to mitigate burnout. This can be done in a number of ways: financially, verbally, furthering their education, and 'including programs that allow them to creatively manage the emotional components of their role and to learn and cultivate life- and practiceenhancing skills. The building of a sense of community in the workplace, in order to cultivate social support networks, has also been also suggested. ${ }^{5}$

Workers with less experience who feel less competent are more likely to leave. 'Managers might avoid turnover if they invest in training and job-related education that increases work-related knowledge and employee self-efficacy. ${ }^{\text {'9 }}$

Other strategies identified in the literature include staff retreats, 4-day work weeks and an increase in staff size. ${ }^{5}$ These strategies are, however, less applicable to the resource-scarce South African environment.

In summary, burnout 'represents an erosion in values, dignity, spirit, and will - an erosion of the human soul' which occurs in areas where job demands exceed the support and resources available to employees. It is not related to people, but rather to the places in which they work, and self-improvement alone will therefore not ameliorate burnout. ${ }^{2}$

\section{Conclusions}

A statistically significant amount of burnout is experienced at RXH. This has a negative effect on the retention of skills and the quality of care at the hospital. For this reason steps must be taken to mitigate the burnout experienced by junior doctors.

These steps can be divided into financially-based and nonfinancially-based factors.

Financially based mitigating factors:

- Recruitment of additional clinical staff (medical officers, SHOs, registrars and consultants)

- More liberal use of temporary staff such as locums

- The introduction of improved productivity aides such as information systems

- Team building activities within departments

- Dedicated training times.

Non-financially based mitigating factors:

- A mentor system with regular sessions for all junior doctors

- $360^{\circ}$ feedback sessions at the end of each rotation

- An orientation programme that allows new junior doctors to 'buddy' with an experienced junior doctor

- Improved communication and transparency between the administration, senior doctors and junior doctors

- Creative shift and leave rosters

- Job sharing

- Exit interviews with junior doctors to ascertain why they are leaving.

\section{References}

. Grant H. From the Transvaal to the Prairies: The migration of South African physicians to Canada. Journal of Ethnic and Migration Studies 2006;32(4):681-695.

Maslach C, Jackson S, Leiter M. Maslach Burnout Inventory Manual. 3rd ed. Palo Alto, CA: Consulting Psychologists, 1996.

3. Richardsen A, Martinussen A. The Maslach Burnout Inventory: Factorial validity and consistency across occupation groups in Norway. Journal of Occupational and Organizational Psychology 2004;77:377-384.

4. Fogarty T, Singh J, Rhoads G, Moore R. Antecedents and consequences of burnout in accounting: Beyond the role stress model. Behavour Research in Accounting 2000;12:31-67.

5. Kalliath T, O'Driscoll M, Gillespie D, Bluedorn A. A test of the Maslach Burnout Inventory in three Kalliath T, O'Driscoll M, Gillespie D, Bluedorn A. A test of the Maslach
samples of healthcare professionals. Work and Stress 2000;14(1):35-50.

6. Garza J, Schneider K, Promecene P, Monga M. Burnout in residency: A statewide study. South Med J
Thes of healthcare professionals. Work and Stress 2000;14(1):35-50. 2000;97(12):1171-1173.

Medland J, Howard-Ruben J, Whitaker E. Fostering psychosocial wellness in oncology nurses: Medland J, Howard-Ruben J, Whitaker E. Fostering psychosocial wellness in oncology
Addressing burnout and social support in the workplace. Oncol Nurs Forum 2004;31(1):47-54.

6. Lert F, Chastang J-F, Castano I. Psychological stress among hospital doctors caring for HIV patients in the late nineties. AIDS Care 2001;13(6):763-778.

. Fogaça M, Carvalho W, Citero V, Norgueira-Martins L. Factors that cause stress for physicians and nurses working in a paediatric and neonatal intensive care unit: Bibliographic review. Revista Brasileira de Terapia Intensiva 2008;20(3):261-266.

8. Barak M, Nissly J, Levin A. Antecedents to retention and turnover among child welfare, social work, and other human service employees: What can we learn from past research? A review and metanalysis. Social Service Review 2001;75(4):625-661.

9. Thanacoody P, Bartram T, Casimir G. The effects of burnout and supervisory social support on the relationship between work-family conflict and intention to leave. Journal of Health Organisation Management 2009;23(1):53-69.

10. Peltzer K, Mashego T, Mabeba M. Short Communication: Occupational stress and burnout among South African medical practitioners. Stress and Health 2003;19:275-280. 